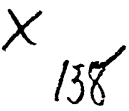

\title{
Über Glukuronsäure und Ätherschwefelsäuren im menschlichen Urine.
}

\author{
Von \\ Dr. C. Tollens.
}

(Aus der städtischen Krankenanstalt in Kiel.

Dirigierender Arzt: Prof. Dr. Hop pe-Seyler.)

(Der Redaktion zugegangen am 1. Juni 1910.)

Im Anschluß an die Veröffentlichungen von mir in Bd. LXI, S. 95, von Stern und mir in Bd. LXIV, S. 39 dieser Zeitschrift möchte ich einige weitere Untersuchungsergebnisse mitteilen. Diese haben sowohl die unter verschiedenen Diätformen und unter pathologischen Verhältnissen ausgeschiedene Menge der Glukuronsäure im menschlichen Urin zum Gegenstande, als sie sich auch besonders mit dem Mengenverhältnis der Glukuronsäure zu den gleichzeitig ausgeschiedenen Ätherschwefelsäuren befassen. Weiter habe ich versucht, einen Einblick zu gewinnen in die Paarungsverhältnisse des reinen per os gegebenen Phenols und Indols zur Glukuronsäure und Schwefelsäure. Da sich wohl dem Phenol die Kresole, dem Indoxyl das Scatoxyl analog verhalten, habe ich mit diesen Substanzen keine weiteren Versuche angestellt.

Die Versuchsanordnung, die sich so von selbst ergab, war folgende: Bei Männern mit gesunden inneren Organen, die geringfügiger äußerer Leiden wegen behandelt wurden, habe ich zunächst bei gemischter Nahrung - einer derben Hausmannskost - die 24 stündige Menge der Glukuronsäure und der Ätherschwefelsäuren des Urins bestimmt. Dann wurde mehrere Tage lang eine sehr reichliche Fett-Fleischdiät gereicht, da hierdurch bekanntlich infolge vermehrter Eiweißfäulnis und vermehrter Bildung aromatischer Produkte im Darme eine Steigerung der Ätherschwefelsäureausscheidung bewirkt wird. Da 
nun schon im normalen Urin an aromatische Fäulnisprodukte gebundene Glukuronsäure neben Ätherschwefelsäure vorkommt, so war es möglich und auch wahrscheinlich, daß sich auch die Glukuronsäure vermehrt, wenn infolge vermehrter Eiweißfäulnis im Darm mehr aromatische Stoffe gebildet werden und in den Urin übergehen. Umsomehr konnte man das erwarten, als überhaupt nur ein Teil der aromatischen Substanzen in der Bindung an Schwefelsäure den Körper verläßt, und als man, wie gesagt, neben der Bindung an Glykokoll und außer dem Auftreten aromatischer Oxysäuren schon längst die Verkuppelung aromatischer Substanzen mit der Glukuronsäure kennt. Baumann, 1) der Entdecker der Ätherschwefelsäure, wies dabei der Glukuronsäure hauptsächlich die Aufgabe zu, erst bei Erschöpfung der paarungsfähigen Schwefelsäure die Entgiftung aromatischer Zersetzungsprodukte resp. eingeführter Gifte durch Paarung mit ihnen vorzunehmen. Spätere Untersuchungen von $\mathrm{Falck}^{2}$ ) und Salkowski ${ }^{3}$ ) wiesen aber auch eine gleichzeitige Paarung aromatischer Substanzen mit Glukuronsäure und Schwefelsäure nach. Falck nimmt, gestützt auf seine Experimente beim Hunde, an, daß selbst dann, wenn recht kleine Mengen Phenol in das Blut und zu den Geweben gelangen, wie es schon normal infolge der Darmfäulnis geschieht, stets ein Teil, bis über $30 \%$, in gepaarte Glukuronsäuren umgewandelt wird. Salkowski betont gelegentlich seiner Untersuchungen über den Stoffwechsel der Herbivoren die gleichzieitige, nebeneinander verlaufende Bildung von gepaarten Glukuronsäuren und Ätherschwefelsäuren.

Nachdem eine Reihe von Versuchen Klarheit in die Ausscheidungsverhältnisse dieser Substanzen bei Fleisch- und Fettkost geschaffen hatte, habe ich dann versucht, durch vorwiegende und auch durch reine Milchkost eine Beschränkung der Fäulnisprozesse im Darme und dementsprechend eine Herabsetzung der im Urin ausgeschiedenen Ätherschwefelsäuren

1) Baumann, u. a. s. Diese Zeitschrift, Bd. III (1879), und Pflügers Archiv f. Physiol., Bd. XIII.

2) Falck, Münch. med. Wochenschr., 1902, S. 1489.

s) E. Salkoẉski, Diese Zeitschrift, Bd. XLII, S. 230 (1904). 
herbeizuführen, um zu sehen, ob diesem Absinken der Ätherschwefelsäuren ein Absinken der Glukuronsäuren parallel gehe. ${ }^{1}$ )

Einige wenige Versuche mit per os gegebenem reinen Indol und Phenol erwiesen sich dann noch als erforderlich zur Klarstellung der Frage, ob und wie sich diese Körper vorzugsweise mit Glukuronsäure oder mit Schwefelsäure paarten. ${ }^{2}$ )

Zunächst lasse ich meine Untersuchungen in Tabellenform folgen:

Aus der Tabelle I geht zunächst hervor, daß bei dem Untersuchten das Verhältnis der Glukuronsäure zur Ätherschwefelsäure sich in der Nähe von 2:1 hält. Nachdem in den oben mitgeteilten Arbeiten mit meiner Methode eine Tagesmenge von

1) Über den Einfluß der Diät auf die Darmfäulnis und Ausscheidung ihrer aromatischen Produkte im Urin siehe unter anderen die Arbeiten:

1. Albu, Deutsche med. Wochenschr., 1887.

2. Baumann, Diese Zeitschrift, Bd. X, S. 123 (1886).

3. Bienstock, Zeitschrift f. klin. Med., Bd. VIII.

4. Biernacki, Arch. f. klin. Med. Bd. XLIX, 1892.

5. Blumenthal, Virchows Archiv, Bd. CXLVI.

6. Hirschler, Diese Zeitschrift, Bd. X, S. 306 (1886).

7. G. Hoppe-Seyler, Diese Zeitschrift, Bd. XII, S. 1 (1888).

8. v. Moraczewski, Zentralbl. f. innere Med. (1909), Nr. 49.

Archiv f. Verdauungskrankheiten, Bd. XIV, H. 4.

9. Fr. Müller, v. Leydens Handbuch der Ernährungstherapie, 1903, 2. Aufl.

10. Poehl, Malys Jahresbericht, 1897.

11. Rovighi, Diese Zeitschrift, Bd. XVI, S. 20 (1891).

12. D. Gerhard, Über Darmfaulnis, Sammelreferat, Ergebnisse der Physiologie, 3. Jg., 1. Abt.

13. v. Noorden, Handbuch der Pathologie des Stoffwechsels.

2) Vgl. dazu die Arbeiten:

1. Austin, Salkowski-Festschrift, Berlin 1904, S. 53.

2. Béla v. Fenyvessy, Malys Jahresb. f. Tierchem., Bd. XXXV, S. 726 (1905).

3. Blumenthal, Bioch. Zeitschr., Bd. I, S. 135 (1906).

4. Falck, s. o.

5. P. Grosser, Diese Zeitschrift, Bd. XLIV.

6. Jaffé, Zentralbl. f. d. med. Wissensch., Bd. I, S. 2 (1872).

7. Külz, Arch. f. d. ges. Physiologie, Bd. XXX, S. 485 (1883).

8. Masson, Arch. de Physiol. norm. et path. (1874), Nr. 2I, S. 760.

9. E. Wang, Diese Zeitschrift, Bd. XXVIII, S. 557. 
Tabelle I. J. (Untersçhenkelgeschwür).

\begin{tabular}{|c|c|c|c|c|c|c|c|}
\hline Tag & $\begin{array}{c}\text { Glukuron- } \\
\text { säure }\end{array}$ & $\begin{array}{c}\text { Äther- } \\
\text { schwefel- } \\
\text { säure }\end{array}$ & $\begin{array}{l}\text { Ver- } \\
\text { hält- } \\
\text { nis }\end{array}$ & Diät & $\begin{array}{c}\text { Dur } \\
\text { Glukuron- } \\
\text { säure }\end{array}$ & $\begin{array}{l}\text { chschnitt } \\
\text { Äther- } \\
\text { schwefel- } \\
\text { säure }\end{array}$ & $\begin{array}{c}\text { Ver- } \\
\text { hält- } \\
\text { nis }\end{array}$ \\
\hline 1 & 0,2730 & 0,162 & $1,7: 1$ & \multirow{2}{*}{$\begin{array}{c}\text { Gemischt } \\
\text {, }\end{array}$} & \multirow{2}{*}{0,3939} & \multirow{2}{*}{0,198} & \multirow{2}{*}{$2: 1$} \\
\hline 2 & 0,5148 & 0,233 & $2,2: 1$ & & & & \\
\hline 3 & 0,6336 & 0,189 & $3,4: 1$ & Fleisch-Fett & \multirow{6}{*}{0,5478} & \multirow{6}{*}{0,277} & \multirow{6}{*}{$2: 1$} \\
\hline 4 & 0,5400 & 0,252 & $2,1: 1$ & , & & & \\
\hline 5 & 0,5996 & 0,310 & $2,0: 1$ & , & & & \\
\hline 6 & $0,42: 36$ & 0,260 & $1,6: 1$ & $>$ & & & \\
\hline 7 & 0,6532 & 0,260 & $2,5: 1$ & , & & & \\
\hline 8 & 0,3888 & 0,190 & $2,0: 1$ & , & & & \\
\hline 9 & 0,4368 & 0,231 & $1,9: 1$ & Milch - Bro & \multirow{5}{*}{0,4792} & \multirow{5}{*}{0,210} & \multirow{5}{*}{$2,3: 1$} \\
\hline 10 & 0,3948 & 0,203 & $2,0: 1$ & , & & & \\
\hline 11 & 0,4050 & 0,151 & $2,7: 1$ & > & & & \\
\hline 12 & 0,4922 & 0,192 & $2,6: 1$ & $\triangleright$ & & & \\
\hline 13 & 0,5670 & 0,273 & $2,1: 1$ & , & & & \\
\hline
\end{tabular}

$0,3-0,4$ g Glukuronsäure im menschlichen Harn als meist vorhanden festgestellt war, konnte man von vornherein bei den allgemein angenommenen Normalwerten von $0,12-0,25 \mathrm{~g}$ Ätherschwefelsäuren im Tagesurin ein mittleres Verhältnis von etwa 2: 1 erwarten. Das Untersuchungsresultat entspricht also der. theoretischen Annahme; allerdings handelt es sich hier um Mittelzahlen aus den untereinander etwas schwankenden Tageswerten. Man wird sich überhaupt über ein starkes Schwanken dieses Verhältnisses nicht wundern dürfen, weil der Wert der Ätherschwefelsäuren schon normalerweise recht schwankt, während der der Glukuronsäure im allgemeinen allerdings gleichmäßiger ist, aber doch auch Schwankungen unterliegt.

Bei der Fleisch-Fettdiät, die etwa $250 \mathrm{~g}$ Eịeiß, $240 \mathrm{~g}$ Fett enthielt, stiegen die Glukuronsäure und zugleich damit die Ätherschwefelsäuren an. Bedeutend ist allerdings weder die Steigerung der Glukuronsäure, noch die der Ätherschwefelsäuren, immerhin übersteigen die Werte sowohl die Normalzahlen wie auch die bei gemischter Kost bei demselben Manne unmittelbar vorher erhaltenen merklich. 
Fett-Fleischdiät steigert also gleichzeitig sowohl die Glukuronsäure als auch die Ätherschwefelsäuren des Urins. Ohne Zweifel ist die gemeinsame Ursache die Vermehrung der Darmfäulnis, deren in den Kreislauf gelangende Produkte gleichzeitig Glukuronsäure und Schwefelsäure zur Paarung heranziehen.

Die Verminderung der Glukuronsäure und der Ätherschwefelsäuren durch Milch-Brotkost - 1,5 l süße Milch, 1 l Sauermilch, $300 \mathrm{~g}$ Brot - gelang in diesem Falle nicht gut. Zwar sank die Menge der Glukuronsäure und der Ätherschwefelsäuren wieder ab bis annähernd zur Norm. Weiter herunter ließen sich aber die Werte durch die 5tägige Milch-Brotnahrung nicht drücken.

Tabelle II. G. (Unterschenkelgeschwür).

Indolversuch.

\begin{tabular}{|c|c|c|c|c|c|c|c|}
\hline Tag & $\begin{array}{c}\text { Glukuron- } \\
\text { säure }\end{array}$ & $\begin{array}{c}\text { Äther- } \\
\text { schwefel- } \\
\text { säuren }\end{array}$ & $\begin{array}{c}\text { Ver- } \\
\text { hält- } \\
\text { nis }\end{array}$ & Diät & $\begin{array}{l}\text { Du } \\
\text { Glukuron- } \\
\text { säure }\end{array}$ & $\begin{array}{l}\text { rchschnitt } \\
\text { Äther- } \\
\text { schwefel- } \\
\text { säuren }\end{array}$ & $\mid \begin{array}{c}\text { Ver- } \\
\text { hält- } \\
\text { nis }\end{array}$ \\
\hline 1 & 0,5607 & 0,277 & $|2,0: 1|$ & \multirow{2}{*}{$\begin{array}{l}\text { Sehr reichlich } \\
\text { Fleisch und Fett }\end{array}$} & \multirow{3}{*}{0,5905} & \multirow{3}{*}{0,257} & \multirow{3}{*}{$2,3: 1$} \\
\hline 2 & 0,5880 & 0,203 & $2,8: 1$ & & & & \\
\hline 3 & 0,8228 & 0,291 & $2,1: 1$ & , & & & \\
\hline 4 & 0,5746 & 0,352 & $|1,6: 1|$ & $\mid$ do. $+0,2$ Indol $\mid$ & $0,5746 !$ & $0,352 !$ & $1,6: 1 !$ \\
\hline 5 & 0,5148 & 0,232 & $2,2: 1$ & Fleisch - Fett & 0,5148 & 0,232 & $2,2: 1$ \\
\hline 6 & 0,4370 & 0,218 & $2,0: 1$ & 1/2 1 Milch & 0 & 2יר 0 & 1 \\
\hline 7 & 0,4590 & 0,248 & $1,9: 1$ & + Gemüse & 0,4400 & $0,2 \cdot 30$ & \\
\hline 8 & 0,4272 & 0,334 & $|1,3: 1|$ & $\mid$ do. $+0,2$ Indol $\mid$ & 0,4272 & 0,334 & $1,3: 1$ ! \\
\hline 9 & 0,5130 & 0,251 & $2,0: 1$ & Gemüse + Milch & 0,5130 & 0,251 & $2,0: 1$ \\
\hline
\end{tabular}

Die Versuchsanordnung erfuhr bei diesem Versuche gegenüber der von Versuch 1 eine wichtige Erweiterung, indem am 4. und 8. Tage je $0,2 \mathrm{~g}$ reinen Indols per os gegeben wurden. Diese Menge wird vom Menschen anstandslos ohne irgend welche krankhaften Erscheinungen vertragen und dürfte, glaube ich, leicht auf $0,5 \mathrm{~g}$ erhöht werden. Wang ${ }^{1}$ ) beobachtete bei einer

1) Wang, s. oben. 
Hündin von mittlerer Größe erst bei $1 \mathrm{~g}$ Zeichen von Nierenreizung. Die Milch-Brotkost des ersten Versuches wurde hier durch eine eiweißarme ersetzt - 1/2 1 Milch und grüne Gemüse -, um ein eventuell bestehendes, direktes Abhängigkeitsverhältnis der Glukuronsäure im Urin vom Eiweißbestand der Nahrung erkennen zu können.

Man sieht, daß das durchschnittliche Verhältnis der Glukuronsäure zur Ätherschwefelsäure sich dem des vorigen Versuches nähert. Wiederum läßt die reichliche Fleisch-Fettdiät die Glukuronsäure und die Ätherschwefelsäuren gleichzeitig ansteigen, wenn auch in geringem Maße. Unter der Wirkung einer eiweißarmen Kost sinkt die Glukuronsäure ein wenig, die Ätherschwefelsäure nimmt kaum ab. Eine sehr wesentliche Verringerung der Darmfäulnis läßt sich also auch hier nicht erzielen, soweit man aus der Glukuronsäure und Ätherschwefelsäure des Urins schließen darf. Ein Abhängigkeitsverhältnis der Glukuronsäure vom Eiweißbestande der Nahrung scheint nicht zu bestehen, denn sonst müßte sicher bei dem geringen Eiweißgehalt der Nahrung von etwa 40 g täglich der Glukuronsäurewert weit tiefer gesunken sein. Damit ist natürlich ein Einblick in einen eventuell vorhandenen Zusammenhang der ausgeschiedenen Glukuronsäuremenge zum Gesamteiweißumsatz im Körper nicht gewonnen. Ein Vergleich zwischen Stickstoffausscheidung und Glukuronsäuremenge würde hier wohl ausschlaggebend sein.

Sehr bemerkenswert ist nun das Verhalten der 0,2 $\mathrm{g}$ Indol. Der in den nächsten 24 Stunden ausgeschiedene Urin wies natürlich alle Merkmale des stark indoxylhaltigen Harns auf. Die Obermayersche Reaktion fiel sehr stark positiv aus. Die Schwefelsäureniederschläge waren direkt blaurot gefärbt. Die Ätherschwefelsäuren waren nun auch, wie zu erwarten, auf die Indoleingabe stark vermehrt, bis auf 0,352 und $0,334 \mathrm{~g}$. Dagegen stieg die Glukuronsäuremenge nicht, d. h. das Indol paarte sich in überwiegendem Maße mit der Schwefelsäure, nicht mit der Glukuronsäure. Für diese kleinen, von mir verwendeten Indolmengen also hat die Baumannsche Ansicht recht, daß in erster Linie die Schwefel- 
säure zur Paarung mit den Fäulnisprodukten des Darms herangezogen werde.

Die naheliegende Schlußfolgerung war anderseits, daß die Glukuronsäure, da sie ja doch nach Tabelle 1 und 2 bei steigender Darmfäulnis parallel den Àtherschwefelsäuren im Urin ansteigt, wenn nicht vom Indol, ihrerseits nun vorzugsweise von Phenolen zur Paarung herangezogen werde. Zur Klärung dieser Frage gab ich im nächsten Falle erst Phenol, dann Indol in Substanz.

Tabelle III. D. (Hallux valgus - operiert).

Indol- und Phenolversuch.

\begin{tabular}{|c|c|c|c|c|c|c|c|}
\hline Tag & $\begin{array}{l}\text { Glu- } \\
\text { kuron- } \\
\text { säure }\end{array}$ & \begin{tabular}{|c|} 
Äther- \\
schwefel- \\
säuren
\end{tabular} & $\begin{array}{c}\text { Ver- } \\
\text { hält- } \\
\text { nis }\end{array}$ & Diät & $\begin{array}{c}\text { Dur } \\
\text { Glukuron- } \\
\text { säure }\end{array}$ & $\begin{array}{l}\text { rchschnitt } \\
\mid \begin{array}{c}\text { Äther- } \\
\text { schwefel- } \\
\text { säuren }\end{array}\end{array}$ & $\begin{array}{c}\text { Ver- } \\
\text { hält- } \\
\text { nis }\end{array}$ \\
\hline 1 & 0,3528 & 0,155 & $2,2: 1$ & Gemischt & 0,3528 & 0,155 & $2,2: 1$ \\
\hline 2 & 0,3840 & 0,135 & $2,9: 1$ & 21 Milch. Stuhl 0 & & & \\
\hline 3 & 0,3699 & 0,191 & $1,9: 1$ & do. Seifeneinlauf & 0,3179 & 0,142 & $2,3: 1$ \\
\hline 4 & 0,3000 & 0,100 & $3,0: 1$ & , öl. Stuhl fest & & & \\
\hline 5 & 0,5000 & 0,150 & $3,3: 1$ & $\times+$ Phenol0,25 & $0,5000 !$ & $0,150 !$ & $3,3: 1$ \\
\hline 6 & 0,3225 & 0,281 & $1,15: 1$ & $\therefore+$ Indol 0,25 & 0,3225 & 0,281 & $1,15: 1$ \\
\hline
\end{tabular}

Die Untersuchung des Falles glückte insofern nicht gut, als bei D. die Milchkost eine sehr hartnäckige Verstopfung nach sich zog, so daß sich auch mit Hilfe energischer Purgantien und von Einläufen kein ausreichender und regelmäßiger Stuhl erzeugen ließ.

Man sieht aber doch wieder, wie der der gemischten Diät entsprechende Normalwert von $0,35 \mathrm{~g}$ Glukuronsäure durch die Milchkost etwas herabgedrückt wird. Gleichzeitig sinken, wenn auch im Durchschnitt nicht viel, die Ätherschwefelsäuren ab. Bei beiden Diätformen das übliche Verhältnis von Glukuronsäure zu Ätherschwefelsäuren von ungefähr $2: 1$.

Auf $0,25 \mathrm{~g}$ Phenol am 5. Tage erfolgt nun eine sehr deutliche Steigerung der Glukuronsäure von 0,3 auf $0,5 \mathrm{~g}$ neben einer verhältnismäßig geringeren der Ätherschwefelsäuren von 0,1 auf $0,15 \mathrm{~g}$. 
0,25 g Indol am nächsten Tage bewirkte dagegen ganz analog dem Fall II ausschließlich eine Steigerung der Ätherschwefelsäuren mit entsprechender Verschiebung der Verhältniszahlen auf $1,15: 1$.

D. h. also, in diesem Falle hat sich die eingeführte kleine Phenolmenge vorzugsweise der Glukuronsäure, das Indol vorzugsweise der Schwefelsäure angelagert. Und ich glaube, daß sich in dieser Form der Satz verallgemeinern läßt. Ich sage dabei absichtlich «vorzugsweise», nicht ausschließlich, denn es gibt im Harn Indoxylglukuronsäure. Erstens ist von HoppeSeyler ${ }^{1}$ ) im Kaninchenharn nach Eingabe einer indoxylbildenden Substanz, - Orthonitrophenylpropiolsäure - von Wang ${ }^{2}$ ) und anderen nach Gaben von reinem Indol im Hundeharn polarimetrisch das Auftreten eines linksdrehenden Körpers festgestellt worden, der der Sachlage nach als Indoxylglukuronsäure angesprochen werden mußte. Zweitens ist sie von P. Mayer und C. Neuberg ${ }^{3}$ ) aus dem menschlichen Urin isoliert und als solche identifiziert worden. Anderseits ist die Vermehrung der Ätherschwefelsäuren, die auch bei meinen Versuchen zwar unbedeutend, aber doch vorhanden war, eine festgestellte Tatsache nach Phenoleingabe. In Vergiftungsfällen kann sogar alle verfügbare Schwefelsäure zur Bindung von Phenolen herangezogen werden, soda $ß$ die Sulfatschwefelsäure aus dem Harne verschwindet. ${ }^{4}$ )

Ein Überblick über die drei Tabellen führt nun zu der Annahme, daß bei den drei untersuchten Personen Phenol und Indol in ziemlich konstantem Verhältnis gebildet wurde. Denn so erklärt sich ungezwungen das gleichzeitige Steigen und Sinken von Glukuronsäure und Ätherschwefelsäuren unter der obigen Voraussetzung, daß Phenol sich vorzugsweise mit Glukuronsäure, Indol mit Schwefelsäure binde.

Die Schwankungen in der Ausscheidung von Glukuron-

1) Hoppe-Seyler, Diese Zeitschrift, Bd. VII, S. 425 (1883).

2) Wang, s. o.

3) P. Mayer und C. Neuberg, Diese Zeitschrift, Bd. XXIX, S. 256 (1900).

4) Wohlgemuth, Berl. klin. Wochenschr., 1906, Nr. 17, u. a. m. 
säure und von Ätherschwefelsäure, wie sie im normalen $\mathrm{Zu}$ stand vorkommen, erklären sich dann wohl aus einer nicht ganz gleichmäßigen Zerlegung des Eiweißes bald unter mehr Bildung von Phenol (aus Tyrosin), bald unter reichlicherer Bildung von Indol (aus Tryptophan).

Ich möchte das Mengenverhältnis beider Substanzen zu einander, ungefähr von $2: 1$, wie es in diesen Fällen war, als das häufigere, normale ansehen. Es gibt aber auch starke Abweichungen davon. Ich konnte nämlich 2 Fälle untersuchen, bei denen das Verhältnis von Glukuronsäure zu Ätherschwefelsäuren in ganz auffälliger Weise zugunsten der Glukuronsäure abwich. Ich fand diese beiden Fälle beim Suchen nach Personen mit niedriger Ätherschwefelsäureausscheidung, die ich am schwachen oder negativen Ausfall der Indoxylreaktion nach Obermayer zu erkennen suchte.

Ich lasse die Tabellen der Untersuchungsresultate folgen.

Tabelle IV. 0. (Unterschenkelgeschwür).

\begin{tabular}{|c|c|c|c|c|c|c|c|c|}
\hline Tag & $\begin{array}{c}\text { Glu- } \\
\text { kuron- } \\
\text { säure }\end{array}$ & \begin{tabular}{|c|} 
Äther- \\
schwefel- \\
säuren
\end{tabular} & $\begin{array}{c}\text { Ober- } \\
\text { mayersche } \\
\text { Reaktion }\end{array}$ & $\begin{array}{c}\text { Ver- } \\
\text { hält- } \\
\text { nis }\end{array}$ & Diät & $\begin{array}{c}\text { Du } \\
\text { Glu- } \\
\text { kuron- } \\
\text { säure }\end{array}$ & $\begin{array}{l}\text { urchschnit } \\
\begin{array}{|}\text { Äther- } \\
\text { schwefel- } \\
\text { säuren }\end{array}\end{array}$ & \begin{tabular}{|l} 
Ver- \\
hält- \\
nis
\end{tabular} \\
\hline 1 & 0,5400 & 0,131 & + & $4,0: 1$ & Gemischt & \multirow{2}{*}{0,5760} & \multirow{2}{*}{0,1350} & \multirow{2}{*}{$4,2: 1$} \\
\hline 2 & 0,6120 & 0,140 & - & $\mathbb{4}, \mathbb{4}: 1$ & , & & & \\
\hline 3 & 0,4536 & 0,206 & $+\underset{\text { sehwach }}{\operatorname{seh}}$ & $2,2: 1$ & $11 \mathrm{sü}$ & \multirow{6}{*}{0,4077} & \multirow{6}{*}{0,117} & \multirow{6}{*}{$3,5: 1$} \\
\hline 4 & 0,3276 & 0,074 & - & $4,4: 1$ & $1 \mathrm{MS}$ & & & \\
\hline 5 & 0,4386 & 0,102 & $+? \underset{\text { schwach }}{\text { gehr }}$ & $4,3: 1$ & & & & \\
\hline 6 & 0,3950 & 0,081 & - & $4,3: 1$ & $\begin{array}{l}\text { reichlic } \\
\text { Zucker }\end{array}$ & & & \\
\hline 7 & 0,4500 & 0,172 & + & $2,7: 1$ & und Brot & & & \\
\hline 8 & 0,3600 & 0,064 & + & $5,6: 1$ & & & & \\
\hline 9 & & 0,054 & + schwach & $10,0: 1$ & Gemischt & \multirow{2}{*}{0,5220} & \multirow{2}{*}{0,148} & \multirow{2}{*}{$3,5: 1$} \\
\hline 10 & & 0,242 & & $2,1: 1$ & s & & & \\
\hline
\end{tabular}

Von vornherein zeichnet sich der Fall vor den drei angeführten aus durch das Überwiegen der Glukuronsäure über die Ätherschwefelsäuren. Denn das Verhaltnis beträgt nicht wie bisher $2: 1$, sondern $4: 1$. Die Menge der Ätherschwefel- 
säure ist dabei höchstens eine mittlere. Die Glukuronsäuremenge aber übersteigt mit $0,54-0,60 \mathrm{~g}$ bei gemischter Diät die mittlere von $0,35 \mathrm{~g}$ bedeutend. Dabei fielen die Indoxylreaktionen zum Teil sehr schwach, zum Teil negativ aus. Man wird also, wenn man nur die Ätherschwefelsäuren berücksichtigen wollte, geneigt sein, eine nur geringe Darmfäulnis anzunehmen, während in Wirklichkeit wohl eine bedeutende vorhanden ist. Nur sind nicht Indoxyl und indoxylbildende Substanzen die Hauptfäulnisprodukte, sondern Phenole, die sich in erster Linie an die Glukuronsäure legen, so ihre Mehrausscheidung bewirkend. Eine zweite Deutung ist in diesem Falle allerdings auch noch möglich mit Berücksichtigung des Umstandes, daß trotz der fäulniswidrigen Milchdiät der Glukuronsäurewert immer relativ hoch bleibt. Man kann daran denken, daß die Glukuronsäure in diesem Falle zum Teil an andere Substanzen gebunden ist, die mit der Darmfäulnis nichts zu tun haben und sonst irgendwo im Stoffwechsel entstehen, vielleicht auch, daß eine unvollkommene Zuckerverbrennung im Sinne P. Mayers ${ }^{1}$ ) vorliegt. Alimentäre Glukosurie ließ sich bei dem Manne nicht erzeugen.

Das starke Überwiegen der Glukuronsäure wird hier auch beibehalten, wenn unter Milch-Brotdiät die gewohnte leichte Verminderung der Glukuronsäure und die auch hier noch etwas unbedeutendere Herabsetzung der Ätherschwefelsäuren eintritt. $\mathrm{Da} ß$ die Milchdiät die Verminderung verursacht, geht wohl sicher aus dem Wiederanstieg von Ätherschwefelsäure und Glukuronsäure am 9. und 10. Tage hervor, wo gemischte, fleisch- und fetthaltige Nahrung gereicht wurde. Auch hier stellte sich übrigens als Folge der Milch-Brotdiät eine äußerst unangenehme, störende Verstopfung ein.

Ich lasse nun noch einen zweiten, ganz analogen Fall folgen, der als Erweiterung des Versuches, so wie Fall 3, Indol und Phenol per os bekam. Außerdem ist er der einzige, bei dem es gelang, durch Milchdiät ein ausgesprochenes $\mathrm{Ab}$ sinken der Glukuronsäure, dagegen nicht der Ätherschwefelsäuren zu erzielen.

1) P. Mayer, Deutsch. med. Wissensch., 1901, Nr. 16 u. 17. 
Tabelle V. K. (Knieverletzung).

Indol- und Phenolversuch.



Auch bei diesem Falle muß der Indoxylgehalt des Harns nach dem negativen Ausfall der Obermayerschen Reaktion sehr gering sein. Dabei erreicht die Tagesmenge der Glukuronsäure außerordentlich hohe Werte, die mit 0,7 und $0,8 \mathrm{~g}$ bei gemischter Diät das Doppelte des Normalen erreichen, während die Ätherschwefelsäuremenge knapp eine mittlere ist.

Besonders bemerkenswert ist nun hier, wie schon erwähnt, daß der Milchgenuß ein beträchtliches Absinken der Glukuronsäure von 0,8 auf $0,26 \mathrm{~g}$ nach sich zog. Dagegen sanken die Ätherschwefelsäuren nicht $a b$, ein Verhalten, das eigentümlich erscheint. Wahrscheinlich war hier der Paarling der Ätherschwefelsäure, nach dem negativen Ausfall der Ob er ma y erschen Reaktion, nur zum kleinsten Teile Indoxyl.

Ganz wie in den vorherigen Fällen war hier die Indolverabreichung von einer beträchtlichen Steigerung der Ätherschwefelsäuren, die Phenolverabreichung von einer beträchtlichen Steigerung der Glukuronsäure gefolgt. Die entsprechende Steigerung der Ätherschwefelsäuren auf Phenol ist verhältnismäßig gering, die der Glukuronsäuren auf Indol ebenfalls und liegt sogar vielleicht noch im Rahmen des Zufälligen, also vom Indol unabhängigen.

An diese Neigung des Indols, sich vorzugsweise mit der Schwefelsäure $\mathrm{zu}$ verbinden und nicht mit der Glukuronsäure, 
ließ sich von vornherein aus folgender Überlegung heraus denken: Ich bestimme die mit Bleiessig und einigen Tropfen Ammoniak ausgefällte Glukuronsäure. Es enthalten aber anscheinend im allgemeinen die Bleisalzfällungen aus menschlichen Urinen sehr wenig oder kein Indoxyl. Jedenfalls gilt dies für die mit Bleiacetat aus saurem Urin gewonnenen Niederschläge. Denn bei verschiedenen quantitativen Bestimmungsmethoden - der von Wang , ${ }^{\text {) }}$ Obermayer, ${ }^{2}$ ) Ellinger ${ }^{3}$ ) - wird der Urin zuerst mit Bleiacetat geklärt, der Niederschlag aber nicht weiter berücksichtigt, da er praktisch frei von Indoxyl iśt. Dagegen lassen sich die Verbindungen der Glukuronsäure im Harne mit Bleisalzen ausfällen. Porcher und Hervieux ${ }^{4}$ ) behaupten auch, daß sich im Harne von Pflanzenfressern (z. B. beim Pferd) Indol nicht mit Glukuronsäure verbinde, da man kein Indol finde in der Bleisalzfällung - P. u. H. verwandten Bleisubacetat! - , die alle Glukuronsäure und jene Substanzen enthalten muß, die sich mit ihr gepaart haben.

Demgegenüber fand Austin ${ }^{5}$ ) im Bleiniederschlage aus menschlichem Urine - mit Bleisubacetat + einigen Tropfen Ammoniak - etwas Indoxyl, das hier nur mit Glukuronsäure verbunden sein konnte. Austin kommt sogar zu der Ansicht, daß die aromatischen Produkte der Darmfäulnis eine größere Verwandtschaft zur Glukuronsäure haben, als zur Schwefelsäure, und daß sie durch Glukuronsäure eher gesättigt werden als durch Schwefelsäure.

Mit dieser künstlichen Beeinflussung der Ausscheidung von Glukuronsäure und Ätherschwefelsäure wäre nun die Ausscheidung beider Substanzen in pathologischen menschlichen Urinen $\mathrm{zu}$ vergleichen.

1) Wang, Diese Zeitschrift, Bd. XXV, S. 406 (1898); Bd. XXVII, S. 135 (1899).

2) Obermayer, Wien. kl. Rundschau, 1898, Nr. 34. Diese Zeitschrift, Bd. XXVI, S. 427 (1899).

3) Ellinger, Diese Zeitschrift, Bd. XXXVIII, S. 178 (1903).

4) Porcher u. Hervieux, Diese Zeitschrift, Bd. XXXIX, S. 147(1904).

5) Salkowski-Festschrift, Berlin 1904, S. 53. 
Man findet fast regelmäßig eine sehr starke Indoxylreaktion in Fällen von Enteritis und Peritonitis tuberculosa. Nach dem vorhergehenden wird man sicher eine entsprechende Vermehrung der Ätherschwefelsäuren zu erwarten haben. Ob eine Vermehrung der Glukuronsäure vorhanden ist, würde weniger vom Indoxyl als von anderen aromatischen Darmfäulnisprodukten, Phenolen in erster Linie, abhängen. Ich untersuchte daraufhin den Harn eines solchen Kranken, der an sehr fortgeschrittenen tuberkulösen Veränderungen litt. Der Urin färbte sich beim Stehen an der Luft von selbst schwärzlich-grün und gab intensiv sämtliche Indoxylreaktionen. Die erhaltenen Werte sind folgende:

$\begin{array}{ccc}\text { Tag } & \text { Ätherschwefelsäure } & \text { Glukuronsäure } \\ 1 & 0,3055 \mathrm{~g} & 0,4452 \mathrm{~g} \\ 2 & 0,3073 \text {, } & 0,7722 \text {; }\end{array}$

Bezüglich der Ätherschwefelsäuren entspricht die Feststellung ihrer Vermehrung der Voraussetzung. Diejenigen aromatischen Stoffe der Darmfäulnis dagegen, die die größere Neigung zur Glukuronsäurepaarung haben, scheinen hier in wechselnder Menge zu entstehen, weil die Glukuronsäure am ersten Tage der normalen, am zweiten einen weit höheren Wert hat. Ihr Verhalten hier kann, wenn man aus einem Falle etwas ersehen darf, insofern auffallend erscheinen, als wir bisher in allen unseren diesbezüglichen Arbeiten beim gesunden Menschen eine größere Gleichmäßigkeit der fortlaufenden Tagesmengen der Glukuronsäuren fanden (vgl. auch S. 141). Im allgemeinen wird man ja eine gleichzeitige und gleichmäßige Vermehrung der Ätherschwefelsäuren und der Glukuronsäure in Fällen pathologischer Darmfäulnis erwarten.

Ferner gehören hierher zwei Fälle von Lysolvergiftung, deren einen ich selbst untersuchen konnte, während ich die Werte des zweiten Herrn Dr. Stern verdanke. Der Urin des ersten schweren Vergiftungsfalles konnte vom Augenblick der Vergiftung an aufgefangen werden. Der zweite Fall wurde erst 7 Stunden nach der Vergiftung eingeliefert; in der Blase befand sich bei der Aufnahme 11 Urin, es ist also die Wahrscheinlichkeit vorhanden, dảß nur wenig oder keiner verloren ging.

Ich lasse die beiden Tabellen folgen: 


\section{Fall.}

Tabelle VI. K. (Vergiftung mit etwa $50 \mathrm{~g}$ Lysol, entsprechend 25 g Kresol.)

\begin{tabular}{c|c|c|c}
\hline \hline Tag & Glukuronsäure & $\begin{array}{c}\text { Äther- } \\
\text { schwefelsäuren }\end{array}$ & $\begin{array}{c}\text { Sulfat- } \\
\text { schwefelsäuren }\end{array}$ \\
\hline 1 & 8,5410 & 1,0815 & 0,2877 \\
2 & 0,3816 & 0,3069 & 3,1017 \\
3 & 0,2970 & 0,1700 & 3,7020 \\
4 & 0,3648 & - & -
\end{tabular}

II. Fall.

Tabelle VII. H. (Vergiftung mit etwa 10-12 g Lysol, entsprechend 5-6 g Kresol.)

\begin{tabular}{c|c|c|c}
\hline Tag & Glukuronsäure & $\begin{array}{c}\text { Äther- } \\
\text { schwefelsäuren }\end{array}$ & $\begin{array}{c}\text { Sulfat- } \\
\text { schwefelsäuren }\end{array}$ \\
\hline 1 & 1,9500 & 0,6392 & - \\
2 & 0,3549 & 0,4453 & -
\end{tabular}

Es handelt sich also um einen schweren und einen leichten Fall von Kresolvergiftung, die sich gegenseitig ergänzen.

Im schweren Falle (Tab. VI) ist fast die gesamte Schwefelsäure bis auf den geringen Rest von $0,2877 \mathrm{~g}$ zur Paarung herangezogen; fast wie im Falle Wohlgemuths, ${ }^{1}$ ) der im Urin nur Ätherschwefelsäuren, aber keine Sulfatschwefelsäure mehr fand.

In viel ausgiebigerem Maße noch hat die Glukuronsäure ihre entgiftende Rolle spielen müssen, indem sie in den ersten 24. Stunden nach der Vergiftung bis $\mathrm{zu} 8,5 \mathrm{~g}$ ansteigt. Um so wichtiger für den Körper ist diese ausgedehnte Möglichkeit der Heranziehung der Glukuronsäure, als in der Regel bei der schweren Kresolvergiftung die Gesamtmenge der ausgeschiedenen Schwefelsäure, wie auch in meinem ersten Falle, gegenüber der Norm stark vermindert ist. Blumenthal ${ }^{2}$ ) hat besonders auf die damit gegebene Beschränkung der Entgiftung durch

1) Wohlgemuth, s. o.

2) Blumenthal, Biochem. Ztschr., Bd. I, S. 135 (1906). 
Schwefelsäure und zugleich auf die außerordentlich große Wichtigkeit der Glukuronsäure als entgiftenden Faktor hingewiesen.

Im zweiten Falle stand am ersten Tage noch Sulfatschwefelsäure zur Entgiftung zur Verfügung. Aber augenscheinlich ist die Bindung des Giftes durch Schwefelsäure und Glukuronsäure gemeinsam schon vollendet gewesen, ehe alle Schwefelsäure verbraucht war. Auffallend erscheint in beiden Fällen der Umstand, daß am zweiten Tage die Glukuronsäure bereits wieder auf ihre normale Menge herabgesunken ist, während die Ätherschwefelsäuren noch relativ stark vermehrt sind.

Ich möchte mich nun nach dem Untersuchungsergebnis dieser beiden Fälle der Ansicht Blumenthals und Salkowskis anschließen, daß bei einer Überschwemmung des Körpers mit Kresol beide Substanzen, sowohl Schwefelsäure als Glukuronsäure, in ganz ausgedehntem Maße zur entgiftenden Paarung herangezogen werden, und das gleichzeitig, und zwar zeigen die Kresole die größere Neigung, sich mit der Glukuronsäure zu paaren, von der dem Körper ein ungleich größerer Vorrat zur Verfügung steht als von Schwefelsäure.

Im wesentlichen ist die Entgiftung bereits nach 24 Stunden (wenn nicht schon viel eher) beendet. In leichten Fällen wird dabei nicht alle Schwefelsäure, die dem Körper zur Verfügung steht, herangezogen - so im Fall 2. In schweren Fällen dagegen kann die Paarung mit Schwefelsäure bis zum Verbrauch der gesamten vorhandenen Schwefelsäure gehen. Damit ist dann aber die weitere Möglichkeit der Paarung beendet, weil eben keine Schwefelsäure mehr da ist. Sehr hoch liegt diese Grenze beim Menschen nicht. Beim Kaninchen ist sie nach Béla v. Fenyvessy ${ }^{1}$ ) bereits nach Eingabe von 0,3 g Phenol erreicht und dann durch höhere Phenoldosen - 0,5 gnicht mehr nach oben zu schieben. Erst Zufuhr von $\mathrm{Na}_{2} \mathrm{SO}_{4}$, also von schwefelsäurebildender Substanz, vermag weitere Paarung und Ausscheidung von Schwefelsäure zu bewirken. Beim Hunde erreichte Blumenthal die Grenze, von der ab die Ätherschwefelsäure im Urin nicht mehr stieg, $\left.{ }^{2}\right)$ mit $1 \mathrm{~g} \mathrm{Lysol}=0,5 \mathrm{~g}$ Kresol.

j) B. v. Fenyvessy, Malys Jahresb., Bd. XXXV, S. 726 (1905).

2) Blumenthal, s. 0 . 
Folgende kurze Berechnung gibt Aufschluß darüber, welchen Phenolmengen im Urin jedesmal die Summe der in meinen beiden Fällen gefundenen Ätherschwefelsäuren und Glukuronsäuren entspricht:

Fall I.

8,5 g Glukuronsäure entsprechen zirka 4,6 g Phenol

1,08 : Ätherschwefelsäure

» $\frac{1,0 \gg>}{5,0}$

Die Summe beider entspricht 5,6 g Phenol.

Da im allgemeinen von einer verschluckten Kresolmenge im Urin 20-25\% wiedererscheinen, und zwar fast alles schon in den ersten 24 Stunden, muß der Urin von Fall 1 etwa 5-6 g Phenole enthalten haben, also eine der rechnerisch gefundenen entsprechende Menge. Nimmt man als Kresolmenge des Urins $5 \mathrm{~g}$, so bleibt auch noch für sonstige Paarlinge genügend Glukuronsäure und Schwefelsäure.

Fall II.

1,95 g Glukuronsäure 0,639 » Ätherschwefelsäure entsprechen zirka 1,1 g Phenol, $\Rightarrow 0,35$.

Die Summe beider entspricht $1,45 \mathrm{~g}$ Phenol.

Zur Resorption müssen in diesem Falle etwa $5-6 \mathrm{~g}$ Kresol gekommen sein, das würde einer Ausscheidung von $1,2-1,5 \mathrm{~g}$ von Phenolen im Urin entsprechen, eine Zahl, die der rechnerisch gefundenen wiederum gut entspricht.

Blumenthal fand in seinen Fällen allerdings Glukuronsäurewerte, welche viel höher waren, als es den Phenolmengen des Urines entsprach. Er nimmt deshalb eine Glukuronsäurebildung im Überschuß an, indem der Körper in seinen Heilbestrebungen über das Ziel hinausschießt. Oder ist vielleicht diese große Anschwemmung von Glukuronsäure als Ausdruck der durch das Kresol gestörten Weiterverbrennung der Glukuronsäure im Sinne P. Mayers ${ }^{1}$ ) zu betrachten? Allerdings sind Blumenthals Werte polarimetrisch gewonnen, also mehr Schätzungswerte. '

1) P. Mayer, s. o.

Hoppe-Seyler's Zeitschrift f. physiol. Chemie. LXVII. 
Die im normalen Urin vorhandene Glukuronsäuremenge von $0,4 \mathrm{~g}$ könnte binden $0,21 \mathrm{~g}$ an Phenolen, also einer Menge, die weit höher ist als die wirklich vorhandene. Denn diese wird nur auf 5-30 mg täglich angegeben. Ein Teil des Überschusses mag nun an Indol gebunden sein, den bei weitem größeren Rest aber müssen andere Paarlinge mit Beschlag belegt haben, die, wie oben angegeben, vielleicht nichts mit Eiweißfäulnis im Darm zu tun haben.

Als Resultat meiner Arbeit ergibt sich folgendes:

1. Der gesunde Mensch scheidet in der Regel mit 0,35 $\mathrm{g}$ täglich doppelt soviel Glukuronsäure aus, als Ätherschwefelsäuren mit $0,18 \mathrm{~g}$ im Mittel. Es kann aber auch sehr viel mehr Glukuronsäure täglich ausgeschieden werden, ohne daß dabei pathologische Verhältnisse vorliegen müßten, während die gleichzeitige Ätherschwefelsäuremenge gering ist.

2. Per os eingeführtes Indol paart sich vorzugsweise mit Schwefelsäure, per os eingeführtes Phenol vorzugsweise mit Glukuronsäure.

3. Ätherschwefelsäuren und Glukuronsäure sinken und steigen parallel mit dem durch Diät beeinflußten Sinken und Steigen der Eiweißfäulnis im Darme meist einander proportional, aber nicht immer; vermutlich je nach dem Verhältnis, in dem bei der Darmfäulnis Phenole und indoxylbildende Substanzen gebildet werden.

4. Pathologischen Steigerungen der Ätherschwefelsäuren - bei Peritonitis und Enteritis tuberculosa - kann eine Steigerung der Glukuronsäure parallel gehen.

5. Bei Kresolvergiftungen werden Glukuronsäure und Schwefelsäure in ausgedehntem Maße zur Entgiftung beide gleichzeitig herangezogen. Vorzüglich aber die Glukuronsäure, zu der die Phenole die größere Verwandtschaft haben. Daher ist meist die Bindung der giftigen Substanzen schon erfolgt, ehe die gesamte Schwefelsäure verbraucht war. In sehr schweren Fällen kann neben großen Glukuronsäuremengen die gesamte Schwefelsäure als Ätherschwefelsäure ausgeschieden werden bis zum Verschwinden der Sulfatschwefelsäure. 